\title{
Duplex selections, equilibrium points, and viability tubes
}

\author{
ZOLTÁN KÁNNAi \\ Department of Mathematics \\ Corvinus University of Budapest, \\ H-1828 Budapest Hungary
}

(Received: October 7, 2015)

\begin{abstract}
Existence of viable trajectories to nonautonomous differential inclusions are proven for time-dependent viability tubes. In the convex case we prove a double-selection theorem and a new Scorza-Dragoni type lemma. Our result also provides a new and palpable proof for the equilibrium form of Kakutani's fixed point theorem.
\end{abstract}

Mathematics Subject Classifications (2015). 34A60, 26E25

Keywords. viable trajectories, sleek tubes, tangential conditions, continuous selections and quasi-selections.

\section{Introduction}

In this paper we examine the viability problem

$$
\left\{\begin{aligned}
x(t) & \in P(t) & & \text { for every } t \\
x^{\prime}(t) & \in F(t, x(t)) & & \text { for a. e. } t \\
x(0) & =x_{0} & &
\end{aligned}\right.
$$

where $P$ is a given map (tube). Viability theorems with a constant viability set are proven by Nagumo [24, Haddad [15], Kánnai and Tallos [19], Aubin, Lygeros, Quincampoix, Sastry, and Seube [3], Gao [11], and recently Gao and Han [12] for autonomous inclusions and by Deimling [9], Ledyaev [23], Tallos 26, and Kánnai and Szabó [17 for nonautonomous inclusions with convex right-hand side; and by Goncharov [14, Colombo [7], and Kánnai and Tallos [18] with nonconvex right-hand side. Nonautonomous viability theorems with a time-dependent tube are proven by Frankowska, Plaskacz and Rzeżuchowski [10], Bothe [5], Gavioli and Malaguti [13], and Kánnai [20] and [21].

A natural extension of the tangential condition for a Caratheodory map $F$ can be written as

$$
F(t, x) \cap D P(t, x)(1) \neq \emptyset \quad \text { for a.e. } t \text { and every } x \in P(t)
$$


in the convex case; while in the nonconvex case,

$$
F(t, x) \subseteq D P(t, x)(1) \quad \text { for a.e. } t \text { and every } x \in P(t),
$$

where $D P(t, x)(1)$ is the contingent derivative to $P$, i.e.

$$
D P(t, x)(1)=\left\{v:(1, v) \in T_{\operatorname{graph} P}(t, x)\right\},
$$

where $T_{\text {graph } P}(t, x)$ is the Bouligand tangent cone to graph $P$ at $(t, x)$ (see e.g. [1]). Note that if $P(t) \equiv K$, then $D P(t, x)(1)=T_{K}(x)$.

Unfortunately, for time-dependent problems condition (2) (and respectively, (3)) is not sufficient for the existence of a viable trajectory. As is well-known, there is a strictly monotone and continuous function $f:[0,1] \rightarrow[0,1]$ such that $f^{\prime}(t)=0$ for a.e. $t$ in $[0,1]$. Then putting $P(t)=\{f(t)\}$ and $F(t, x) \equiv\{0\}$, condition (3) is valid, $P$ has a compact graph, however, it does not admit a viable solution to $(1)$.

We will see that if the tube $P$ is sleek (see below), then the condition

$$
D P(t, x)(1) \neq \emptyset \quad \text { for every }(t, x) \in \operatorname{graph} P
$$

combined with (2) (or respectively, (3)) is already sufficient to guarantee the existence of viable solutions. Note that the tangential condition (2) alone yields $D P(t, x)(1) \neq \emptyset$ for a.e. $t$ and every $x \in P(t)$. However, this property is quite far from (4) because, as shown by the previous example, solutions may not exist. Even if $P$ is sleek, (2) will not be sufficient, either, because the map $P:[0,2] \rightarrow \mathcal{P}(\mathbb{R})$

$$
P(t)= \begin{cases}\left\{1-\sqrt{1-t^{2}}\right\} & \text { if } 0 \leq t<1 \\ {[1,3]} & \text { if } t=1 \\ \left\{3+\sqrt{1-(t-2)^{2}}\right\} & \text { if } 1<t \leq 2\end{cases}
$$

is sleek, has a compact graph, and even (4) is fulfilled except only one point, however $P$ has no absolutely continuous selection.

In 20] we examined viability on sleek tubes, but under virtually stronger conditions than (4). Seeing the above examples, it is expectable (as e. g. in [10]) that a condition guaranteeing the viability of a tube, will be strong enough, e. g. essentially the tube shall be absolutely continuous. But, one of our purposes in viability theory, is to weaken the virtual tangential condition. In this work, we give viability theorems on sleek tubes with only (4), moreover in the convex case we retain upper semicontinuity in the second variable, in contrast to the parallel results in 20 and [10]. In the convex case, Bothe 5 proved a somewhat stronger result in finite dimension exploiting a different technique. Nevertheless, 5] uses a skilled definition (almost u.s.c. maps). Our Scorza-Dragoni-type lemma retains the classical conditions for the right-hand side: measurability, and upper semicontinuity in the second variable. The significance of this lemma is, that the values of $F$ are unchanged on a "big" set, hence the tangential condition remains valid there. Using the classical result [25], we cannot guarantee the tangential condition for the regularized map. The tools applied by us seem to be useful also in equilibrium theory, as we will see in the final paragraph. 


\section{Preliminaries}

In the following let $X$ be a Banach space.

Definition $1 A$ set $K \subseteq X$ is said to be sleek if its Bouligand cone map

$$
T_{K}: K \rightarrow \mathcal{P}(x), \quad x \mapsto T_{K}(x)
$$

is lower semicontinuous (l.s.c.). A map $P:[0,1] \rightarrow \mathcal{P}(X)$ is called sleek if graph $P$ is a sleek subset of $\mathbb{R} \times X$. $P$ is called properly sleek if it is sleek moreover (4) is fulfilled for every $0 \leq t<1$ and $x \in P(t)$. Finally, $P$ is called strongly sleek if it is sleek, and there is a constant $M>0$ such that

$$
D P(t, x)(1) \cap M \cdot B \neq \emptyset \text { for every }(t, x) \in \operatorname{graph} P \cap([0,1) \times X) .
$$

We remark that if $K$ is sleek, then for every $x \in K, T_{K}(x)=C_{K}(x)$ (where $C_{K}(x)$ is the Clarke cone to $K$ at $x$, see e.g. [1]), thus, $T_{K}(x)$ is convex and closed. Consequently, whenever $P$ is a sleek map,then the sets $D P(t, x)(1)$ are also convex and closed.

In [20] the following theorem is proven:

Theorem 1 Suppose that $X$ is reflexive. Let $P:[0,1] \rightarrow \mathcal{P}(X)$ be a strongly sleek map with a compact graph, $F$ : graph $P \rightarrow \mathcal{P}(X)$ be a $\Lambda \otimes \mathcal{B}$-measurable, integrably bounded and nonempty closed valued map, which is l.s.c. in $x$, and assume (3) is valid. Then there is a function $x \in A C_{X}[0,1]$ satisfying (1).

Now we verify two lemmata.

Lemma 1 Let $P:[0,1] \rightarrow \mathcal{P}(X)$ be a sleek map. Then $D P(\cdot, \cdot)(1)$ is l.s.c. on its domain. Consequently, if $P$ is properly sleek, then

$$
D P(\cdot, \cdot)(1): \operatorname{graph} P \cap([0,1) \times X) \rightarrow \mathcal{P}(X)
$$

is a nonemty closed and convex valued l.s.c. map.

Proof. Take an open set $H \subseteq X$ such that for some $\left(t_{0}, x_{0}\right) \in \operatorname{graph} P$,

$$
D P\left(t_{0}, x_{0}\right)(1) \cap H \neq \emptyset .
$$

Taking a point $v \in D P\left(t_{0}, x_{0}\right)(1) \cap H$ there is a number $\frac{1}{2}>\varepsilon>0$ such that $v+\varepsilon \cdot B^{\mathrm{o}} \subseteq H$, on the other hand, since $P$ is sleek, there is a neighborhood $U$ of $\left(t_{0}, x_{0}\right)$ in graph $P$ such that

$$
\left(1-\frac{\varepsilon}{4\|v\|+4}, 1+\frac{\varepsilon}{4\|v\|+4}\right) \times\left(v+\frac{\varepsilon}{2} \cdot B^{\circ}\right) \cap T_{\operatorname{graph} P}(t, x) \neq \emptyset
$$

for an arbitrary fixed $(t, x) \in U$. Then there exist $\frac{3}{2}>\alpha>\frac{1}{2}$ and $u \in v+\frac{\varepsilon}{2} \cdot B^{\text {o }}$ such that $(\alpha, u) \in T_{\operatorname{graph} P}(t, x)$, thus, $\left(1, \frac{u}{\alpha}\right) \in T_{\operatorname{graph} P}(t, x)$, hence

$$
\frac{u}{\alpha} \in D P(t, x)(1) .
$$


At the same time,

$$
\left\|\frac{u}{\alpha}-v\right\| \leq \frac{|\alpha-1|}{\alpha} \cdot\|u\|+\|u-v\|<\frac{\varepsilon \cdot(\|v\|+1)}{2\|v\|+2}+\frac{\varepsilon}{2}=\varepsilon,
$$

hence $\frac{u}{\alpha} \in H$, consequently

$$
D P(t, x)(1) \cap H \neq \emptyset
$$

for any $(t, x) \in U$. Thus, $D P(\cdot, \cdot)(1)$ is l.s.c..

Lemma 2 Let $P:[0,1] \rightarrow \mathcal{P}(X)$ be a properly sleek map with a compact graph. Then for every number $0<T<1, P$ is strongly sleek on $[0, T]$.

Proof. For every $n \in \mathbb{N}$, the set

$$
G_{n}=\left\{(t, x) \in \operatorname{graph} P \cap([0,1) \times X): D P(t, x)(1) \cap n \cdot B^{\circ} \neq \emptyset\right\}
$$

is open subset of graph $P$ in accordance with the previous lemma. Hence $\left(G_{n}\right)_{n \in \mathbb{N}}$ is an open covering of the compact set graph $P \cap([0, T] \times X)$. Then there is an integer $n$ such that

$$
\text { graph } P \cap([0, T] \times X) \subseteq G_{n},
$$

which just means that $P$ is strongly sleek on $[0, T]$.

\section{Viability. A double-selection theorem.}

First consider the nonconvex case.

Theorem 2 Suppose $X$ is reflexive. Let $P:[0,1] \rightarrow \mathcal{P}(X)$ be a properly sleek map with a compact graph, $F$ : graph $P \rightarrow \mathcal{P}(X)$ be a $\Lambda \otimes \mathcal{B}$-measurable, integrably bounded and nonempty closed valued map, which is l.s.c. in the second variable. If the tangential condition (3) is valid for a.e. $t$ and every $x \in P(t)$, then (1) has a solution $x \in A C_{X}[0,1]$ for any $x_{0} \in P(0)$.

Proof. Take a strictly monotone sequence $\left(T_{n}\right)$ from $[0,1]$ converging to 1 such that $T_{0}=0$. According to Lemma $2, P$ is strongly sleek on every $\left[T_{n}, T_{n+1}\right]$. By an inductive way, using Theorem 1 we define the function $x$ on every $\left[T_{n}, T_{n+1}\right]$ so that $x$ can satisfy (1) on $\left[T_{n}, T_{n+1}\right]$ in the meaning that in every step the initial condition coincides with the final data of the preceding step. Then $x$ is absolutely continuous on every $\left[T_{n}, T_{n+1}\right]$ and $\left\|x^{\prime}(t)\right\| \leq \ell(t)$ for a.e. $t \in[0,1]$, where $\ell \in L^{1}[0,1]$ is an integrable bound of $F$. Hence $x$ is obviously absolutely continuous on $[0,1]$ and satisfies $(1)$ on $[0,1]$.

The convex case is technically more involved than the nonconvex one. For this we prove a double-selection theorem, which can be considered as the unification of theorems of Michael and Cellina [2].

In the following, fix a compact metric space $K$, a Banach space $X$ and a closed convex subset $Y \subseteq X$.

The next proposition is easy to verify (and also well-known). 
Proposition 1 Let $r$ be a positive number, $F: K \rightarrow \mathcal{P}(X)$ be a nonempty valued l.s.c. map, and $f: K \rightarrow X$ be a continuous function such that

$$
d(f(x), F(x))<r
$$

for every $x \in K$. Then the map $G: K \rightarrow \mathcal{P}(X)$,

$$
G(x)=F(x) \cap\left(f(x)+r \cdot B^{\mathrm{o}}\right)
$$

is also l.s.c.

We will need a technical lemma.

Lemma 3 Let $F: K \rightarrow \mathcal{P}(Y)$ and $G: K \rightarrow \mathcal{P}(X)$ be nonempty convex valued maps such that

$$
F(x) \cap G(x) \neq \emptyset
$$

for every $x \in K$. Suppose that $F$ is Hausdorff-u.s.c. (H-u.s.c.) and $G$ is l.s.c. Then for arbitrary $\varepsilon>0$ there is a continuous function $f_{\varepsilon}: K \rightarrow Y$ such that

$$
\operatorname{graph} f_{\varepsilon} \subseteq B(\operatorname{graph} F, \varepsilon)
$$

and for every $x \in K$,

$$
f_{\varepsilon}(x) \in G(x)+\varepsilon \cdot B .
$$

Proof. For every $x \in K$ fix a vector

$$
v_{x} \in F(x) \cap G(x) .
$$

Take a number $\varepsilon>0$. Then $F$ being H-u.s.c. and $G$ being l.s.c., every point $x \in K$ has an open neighborhood $B^{\circ}\left(x, \delta_{x}\right)$ with radius $0<\delta_{x}<\varepsilon$ such that

$$
F(y) \subseteq F(x)+\varepsilon \cdot B,
$$

and

$$
G(y) \cap\left(v_{x}+\varepsilon \cdot B\right) \neq \emptyset
$$

for every $y \in B^{\circ}\left(x, \delta_{x}\right)$. Then

$$
\left\{B^{\circ}\left(x, \frac{\delta_{x}}{2}\right): x \in K\right\}
$$

is an open covering of $K$, hence there are points $x_{1}, x_{2}, \ldots, x_{n} \in K$ such that

$$
K=\bigcup_{k=1}^{n} B^{\circ}\left(x_{k}, \frac{\delta_{x_{k}}}{2}\right) \text {. }
$$

For every $1 \leq k \leq n$, consider the distance functions

$$
\varphi_{k}=d\left(\cdot,\left[B^{\circ}\left(x_{k}, \frac{\delta_{x_{k}}}{2}\right)\right]^{c}\right) .
$$


Since the sets $B^{\circ}\left(x_{k}, \frac{\delta_{x_{k}}}{2}\right)$ cover $K$, for any $x \in K$,

$$
\sum_{j=1}^{n} \varphi_{j}(x)>0
$$

Denote for every $1 \leq k \leq n$,

$$
\psi_{k}=\frac{\varphi_{k}}{\sum_{j=1}^{n} \varphi_{j}}
$$

Then each $\psi_{k}$ is a nonnegative continuous function, furthermore

$$
\sum_{k=1}^{n} \psi_{k}(x)=1
$$

for every $x \in K$. Then

$$
f_{\varepsilon}(x)=\sum_{k=1}^{n} \psi_{k}(x) \cdot v_{x_{k}}
$$

being a convex combination of some elements of $Y$, we have that $f_{\varepsilon}(x) \in Y$. So the latest equality defines a continuous function $f_{\varepsilon}: K \rightarrow Y$. We will show that $f_{\varepsilon}$ satisfies the statement. Fix a point $x \in K$. Note that

$$
x \in B^{\circ}\left(x_{k}, \frac{\delta_{x_{k}}}{2}\right) \quad \text { i.e. } \quad x_{k} \in B^{\circ}\left(x, \frac{\delta_{x_{k}}}{2}\right)
$$

whenever $\psi_{k}(x)>0$. Now consider all indexes $1 \leq k \leq n$ such that $\psi_{k}(x)>0$. Among them, there is an index for which $\delta_{x_{k}}$ is maximal; thus,

there exists a number $1 \leq p \leq n$ such that

- $\psi_{p}(x)>0$, consequently $d\left(x, x_{p}\right)<\frac{\delta_{x_{p}}}{2}$;

- whenever $1 \leq k \leq n, \psi_{k}(x)>0$, then $\delta_{x_{k}} \leq \delta_{x_{p}}$; hence $d\left(x, x_{k}\right)<\frac{\delta_{x_{k}}}{2}$.

Furthermore by

$1=\sum_{k=1}^{n} \psi_{k}(x)=\sum_{\psi_{k}(x)>0} \psi_{k}(x)$ and $f_{\varepsilon}(x)=\sum_{k=1}^{n} \psi_{k}(x) \cdot v_{x_{k}}=\sum_{\psi_{k}(x)>0} \psi_{k}(x) \cdot v_{x_{k}}$

$f_{\varepsilon}(x)$ appears as a convex combination of vectors $v_{x_{k}}$ such that $\psi_{k}(x)>0$. At the same time, for every such index $k$ we have

$$
d\left(x_{k}, x_{p}\right) \leq d\left(x_{k}, x\right)+d\left(x, x_{p}\right)<\frac{\delta_{x_{k}}}{2}+\frac{\delta_{x_{p}}}{2} \leq \delta_{x_{p}}
$$

consequently

$$
x_{k} \in B^{\circ}\left(x_{p}, \delta_{x_{p}}\right),
$$


hence on the one hand

$$
F\left(x_{k}\right) \subseteq F\left(x_{p}\right)+\varepsilon \cdot B,
$$

so $v_{x_{k}}$ belongs to the convex set $F\left(x_{p}\right)+\varepsilon \cdot B$; on the other hand,

$$
f_{\varepsilon}(x) \in F\left(x_{p}\right)+\varepsilon \cdot B
$$

since $f_{\varepsilon}(x)$ is a convex combination these aforementioned vectors $v_{x_{k}}$. So there is a vector $v \in F\left(x_{p}\right)$ such that $\left\|v-f_{\varepsilon}(x)\right\|<\varepsilon$, and $d\left(x, x_{p}\right)<\delta_{x_{p}}<\varepsilon$, hence

$$
\left(x, f_{\varepsilon}(x)\right) \in B(\operatorname{graph} F, \varepsilon) .
$$

Moreover for each index $k$ satisfying $\psi_{k}(x)>0$ we have

$$
G(x) \cap\left(v_{x_{k}}+\varepsilon \cdot B\right) \neq \emptyset
$$

since $x \in B^{\circ}\left(x_{k}, \frac{\delta_{x_{k}}}{2}\right)$. Hence

$$
v_{x_{k}} \in G(x)+\varepsilon \cdot B
$$

for all these $k$, thus, $G(x)+\varepsilon \cdot B$ being convex, we get that

$$
f_{\varepsilon}(x) \in G(x)+\varepsilon \cdot B,
$$

which just means the statement of the lemma.

Note that the previous lemma obviously implies the approximate selection theorem of Cellina. By applying Lemma 3 to the map $G(x)=F(x) \cap$ $\left(f_{0}(x)+r \cdot B^{\circ}\right)$ and the constant map valued $Y$, we obtain the following:

Corollary 1 Let $F: K \rightarrow \mathcal{P}(X)$ be a nonempty convex valued l.s.c. map, $r$ be a positive number, and $f_{0}: K \rightarrow Y$ be a continuous function such that

$$
f_{0}(x) \in F(x)+r \cdot B
$$

for every $x \in K$. Then for every number $\varepsilon>0$ there is a continuous function $f_{\varepsilon}: K \rightarrow Y$ such that

1. $f_{\varepsilon}(x) \in F(x)+\varepsilon \cdot B$ for all $x \in K$;

2. $\left\|f_{\varepsilon}(x)-f_{0}(x)\right\|<r+\varepsilon$ for all $x \in K$.

LEMMA 4 Let $F: K \rightarrow \mathcal{P}(X)$ be a nonempty closed convex valued l.s.c. map, $r$ be a positive number, and $f_{0}: K \rightarrow Y$ be a continuous function such that

$$
f_{0}(x) \in F(x)+r \cdot B
$$

for every $x \in K$. Then there exists a continuous function $f: K \rightarrow Y$ such that for every $x \in K$,

$$
f(x) \in F(x) \quad \text { and } \quad\left\|f(x)-f_{0}(x)\right\| \leq 2 r \text {. }
$$


Proof. Define a sequence of continuous functions $f_{n}: K \rightarrow Y$, by induction.

- By Corollary 1 choose a continuous function $f_{1}: K \rightarrow Y$ such that $f_{1}(x) \in$ $F(x)+\frac{r}{2} \cdot B$ and $\left\|f_{1}(x)-f_{0}(x)\right\|<r+\frac{r}{2}$ for every $x \in K$.

- When $f_{n}$ is already defined, and $f_{n}(x) \in F(x)+\frac{r}{2^{n}} \cdot B$ for every $x \in K$, then applying Corollary 1 again, choose a continuous function $f_{n+1}: K \rightarrow$ $Y$ such that $f_{n+1}(x) \in F(x)+\frac{r}{2^{n+1}} \cdot B$ and $\left\|f_{n+1}(x)-f_{n}(x)\right\|<\frac{r}{2^{n}}+\frac{r}{2^{n+1}}$ for every $x \in K$.

By all these we have defined the sequence $\left(f_{n}\right)$. One can immediately see that this sequence uniformly converges to a continuous function $f: K \rightarrow Y$. Since

$$
d\left(f_{n}(x), F(x)\right) \leq \frac{r}{2^{n}}
$$

for every $x \in K$ and $n \in \mathbb{N}$, we obviously get that

$$
d(f(x), F(x))=0, \quad \text { i.e. } \quad f(x) \in F(x)
$$

for every $x \in K$. Furthermore

$$
\left\|f_{n}(x)-f_{0}(x)\right\| \leq \sum_{k=1}^{n}\left\|f_{k}(x)-f_{k-1}(x)\right\| \leq \sum_{k=1}^{n} \frac{r}{2^{k-1}} \leq 2 r
$$

for every $x \in K$ and $n \in \mathbb{N}$, thus, taking the limit we have

$$
\left\|f(x)-f_{0}(x)\right\| \leq 2 r
$$

Now we arrived at the main technical results.

TheOREM 3 Let $F: K \rightarrow \mathcal{P}(Y)$ and $G: K \rightarrow \mathcal{P}(X)$ nonempty convex valued maps such that

$$
F(x) \cap G(x) \neq \emptyset
$$

for every $x \in K$, moreover $F$ is $H$-u.s.c. and és $G$ closed valued l.s.c.. Then for every $\varepsilon>0$ there is a continuous function $f_{\varepsilon}: K \rightarrow Y$ such that

$$
\operatorname{graph} f_{\varepsilon} \subseteq B(\operatorname{graph} F, \varepsilon)
$$

and

$$
f_{\varepsilon}(x) \in G(x)
$$

for every $x \in K$.

Proof. According to Lemma 3, there is a continuous function $f_{*}: K \rightarrow Y$ such that

$$
\operatorname{graph} f_{*} \subseteq B\left(\operatorname{graph} F, \frac{\varepsilon}{4}\right),
$$


moreover for every $x \in K$,

$$
f_{*}(x) \in G(x)+\frac{\varepsilon}{4} \cdot B .
$$

Then by Lemma 4 , there is a continuous function $f_{\varepsilon}: K \rightarrow Y$ such that

$$
f_{\varepsilon}(x) \in G(x)
$$

and

$$
\left\|f_{\varepsilon}(x)-f_{*}(x)\right\| \leq \frac{\varepsilon}{2}
$$

for every $x \in K$. Hence we obviously get that

$$
\operatorname{graph} f_{\varepsilon} \subseteq B(\operatorname{graph} F, \varepsilon) \text {. }
$$

The following statement is a new version of the celebrated Scorza-Dragoni lemma.

LEMMA 5 Let $T$ be a compact subset of $\mathbb{R}, X$ and $Y$ be separable metric spaces, $P: T \rightarrow \mathcal{P}(X)$ be a compact valued Lebesgue-measurable map, and finaly $F$ : graph $P \rightarrow \mathcal{P}(Y)$ be a compact valued $\Lambda \otimes \mathcal{B}$-measurable map, which is u.s.c. in the second variable. Then for every $\varepsilon>0$, there is a compact subset $A_{\varepsilon} \subseteq T$ such that the Lebesgue measure of $T \backslash A_{\varepsilon}$ is less than $\varepsilon$, and the restriction of $F$ to graph $P \cap\left(A_{\varepsilon} \times X\right)$ has a compact graph.

Proof. graph $F$ is obviously $\Lambda \otimes \mathcal{B}_{X \times Y}$-measurable. Then there is a zero measured Borel set $H \subseteq T$ such that graph $F \backslash H \times X \times Y$ is $\mathcal{B}_{1} \otimes \mathcal{B}_{X \times Y}$-measurable. Put $\Phi: T \rightarrow \mathcal{P}(T \times X \times Y)$,

$$
\Phi(t)=\left[(\operatorname{graph} F \backslash H \times X \times Y) \cup H \times\left\{\left(x_{0}, v_{0}\right)\right\}\right] \cap(\{t\} \times X \times Y),
$$

with an arbitrary $\left(x_{0}, v_{0}\right) \in X \times Y$. Since $P$ and $F$ are compact valued and $F$ is u.s.c. in the second variable, the values of $\Phi$ are compact. On the other hand,

$\operatorname{graph} \Phi=\left(\Delta_{T} \times X \times Y\right) \cap\left(T \times\left[(\operatorname{graph} F \backslash H \times X \times Y) \cup H \times\left\{\left(x_{0}, v_{0}\right)\right\}\right]\right)$

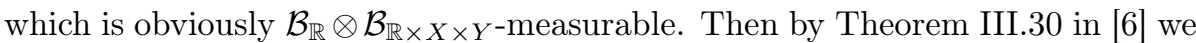
have that $\Phi$ is a Lebesgue-measurable map. Applying Lusin's theorem (in the metric space of compact subsets in $T \times X \times Y$ with the Hausdorff distance) we get that for any $\varepsilon>0$, there is a compact subset $A_{\varepsilon} \subseteq T$ with $\lambda\left(T \backslash A_{\varepsilon}\right)<\varepsilon$ such that the restriction of $\Phi$ is Hausdorff-continuous. We can obviously suppose that $H \subseteq A_{\varepsilon}$. Then the compactness of

$$
\bigcup_{t \in A_{\varepsilon}} \Phi(t)=\operatorname{graph}\left(F_{\mid \operatorname{graph}} P \cap\left(A_{\varepsilon} \times X\right)\right)
$$

follows our statement.

Let us now return to viability. 
Proposition 2 Suppose that $X$ is a separable Hilbert space and $Y \subseteq X$ is convex, bounded and closed. Let $P:[0,1] \rightarrow \mathcal{P}(X)$ be a properly sleek map with a compact graph, $F$ : graph $P \rightarrow \mathcal{P}(X)$ be a closed and convex valued $\Lambda \otimes \mathcal{B}$-measurable map, which is u.s.c. in the second variable, moreover

$$
F(t, x) \subseteq Y
$$

and the tangential condition (2) is valid for a.e. $t \in[0,1]$ and every $x \in P(t)$. Then (1) has a solution $x \in A C_{X}[0,1]$ for any $x_{0} \in P(0)$.

Proof. At first we show the existence to $(1)$ on $[S, T]$ with an arbitrary $x_{0} \in$ $P(S)$ where $0 \leq S<T<1$. By Lemma 2, $P$ is strongly sleek on $[S, T]$ with a constant $M>d(0, Y)+\operatorname{diam} Y+1$. Then the map

$$
G:(t, x) \mapsto \overline{D P(t, x)(1) \cap M \cdot B^{\circ}}
$$

is l.s.c. on graph $P \cap([S, T] \times X)$ moreover

$$
F(t, x) \cap G(t, x) \neq \emptyset
$$

for a.e. $t \in[S, T]$ and every $x \in P(t)$. Fix a number $n \in \mathbb{N}$. Then by Lemma 5 , we get that there is an compact set $A_{n} \subseteq[S, T]$ such that the Lebesgue measure of $[S, T] \backslash A_{n}$ is less than $\frac{1}{n}$, moreover $F$ on graph $P \cap\left(A_{n} \times X\right)$ has a compact graph and satisfies (6). Denote by $F_{n}$ this restricted map. Then $F_{n}$ is u.s.c.. Now by applying Theorem 3 , we have a continuous function

$$
f_{n}: \operatorname{graph} P \cap\left(A_{n} \times X\right) \rightarrow Y
$$

such that

$$
\operatorname{graph} f_{n} \subseteq B\left(\operatorname{graph} F_{n}, \frac{1}{n}\right),
$$

and $f_{n}(t, x) \in G(t, x)$ for every $(t, x) \in$ graph $P \cap\left(A_{n} \times X\right)$. Since $G$ is a closed and convex valued l.s.c. map, by Michael's selection theorem $f_{n}$ can be continuously extended to graph $P \cap([S, T] \times X)$ so that $f_{n}(t, x) \in G(t, x)$, i.e.

$$
f_{n}(t, x) \in D P(t, x)(1) \quad \text { and } \quad\left\|f_{n}(t, x)\right\| \leq M
$$

for every $(t, x) \in \operatorname{graph} P \cap([S, T] \times X)$. Then by applying Theorem 4.4.1 in [2], we have that there is a function $x_{n} \in A C_{X}[S, T]$ such that

$$
\begin{cases}x_{n}(t) \in P(t) & \text { for every } t \in[S, T] \\ x_{n}^{\prime}(t)=f_{n}\left(t, x_{n}(t)\right) & \text { for a. e. } t \\ x_{n}(S)=x_{0} & \text {. }\end{cases}
$$

We can obviously suppose that the sets $A_{n}$ and graph $F_{n}$ increase in $n$. Now for a.e. $t \in[S, T]$ and every $n,\left\|x_{n}^{\prime}(t)\right\| \leq M$, so the sequence $\left(x_{n}^{\prime}\right)$ has a weakly convergent subsequence (denoted by $\left(x_{n}^{\prime}\right)$ again) in $L_{X}^{2}[S, T]$, converging to a function $u \in L_{X}^{2}[S, T]$. Define

$$
x:=x_{0}+\int_{S} u .
$$


Then $x^{\prime}=u$, i.e. $x_{n}^{\prime} \rightarrow x^{\prime}$ weakly in $L_{X}^{2}[S, T]$, moreover for every $S \leq t \leq T$, $x_{n}(t)$ tends to $x(t)$ weakly in $X$. Thus, by compactness of $P(t)$ we get that $x_{n}(t)$ tends to $x(t)$ in norm and $x(t) \in P(t)$ for every $t \in[S, T]$. By definition $x(S)=x_{0}$. Now fix $n \in \mathbb{N}$. Then for every $k \geq n$ and a.e. $t \in A_{n}$, obviously

$$
\left(t, x_{k}(t), x_{k}^{\prime}(t)\right) \in B\left(\operatorname{graph} F_{n}, \frac{1}{k}\right),
$$

so by the Kuratowski-Ryll-Nardzewski selection theorem (see [6]) the measurable map

$$
t \mapsto \Gamma_{k}(t)=\left[\left(t, x_{k}(t), x_{k}^{\prime}(t)\right)+\frac{2}{k} \cdot B\right] \cap \operatorname{graph} F_{n}
$$

has a measurable selection $t \mapsto\left(u_{k}(t), v_{k}(t), w_{k}(t)\right)$. Then necessarily, $u_{k}(t) \rightarrow$ $t, v_{k}(t) \rightarrow x(t)$ for a.e. $t \in A_{n}$, and $w_{k} \rightarrow x^{\prime}$ weakly in $L_{X}^{2}[S, T]$, moreover

$$
w_{k}(t) \in F_{n}\left(u_{k}(t), v_{k}(t)\right)
$$

for a.e. $t \in A_{n}$. Then for any $k \geq m \geq n$,

$$
w_{k}(t) \in \overline{\operatorname{conv}} \bigcup_{j=m}^{+\infty} F_{n}\left(u_{k}(t), v_{k}(t)\right),
$$

on the other hand, all measurable selections of the right-hand side (as a map of $t$ ) appears as a weakly closed subset of $L_{X}^{2}[S, T]$. So

$$
x^{\prime}(t) \in \overline{\operatorname{conv}} \bigcup_{j=m}^{+\infty} F_{n}\left(u_{k}(t), v_{k}(t)\right)
$$

for a.e. $t \in A_{n}$ and every $m \geq n$, hence we easily get that

$$
x^{\prime}(t) \in \bigcap_{m=1}^{+\infty} \overline{\operatorname{conv}} \bigcup_{j=m}^{+\infty} F_{n}\left(u_{k}(t), v_{k}(t)\right) \subseteq F_{n}(t, x(t)) \subseteq F(t, x(t))
$$

for a.e. $t \in A_{n}$. Since it is true for any $n$, then $x^{\prime}(t) \in F(t, x(t))$ for a.e. $t \in[S, T]$. Now the rest of the proof is carried out similarly to Theorem 2 .

Theorem 4 Let $X$ be a Banach space, $P:[0,1] \rightarrow \mathcal{P}(X)$ be a properly sleek map with a compact graph, $F:$ graph $P \rightarrow \mathcal{P}(X)$ be a weakly compact and convex valued $\Lambda \otimes \mathcal{B}$-measurable and integrably bounded map, which is u.s.c. in the second variable with respect to the norm topology in graph $P$ and the weak topology in $X$, moreover the tangential condition $(2)$ is valid for a.e. $t \in[0,1]$ and every $x \in P(t)$. Then (1) has a solution defined on $[0,1]$ for any $x_{0} \in P(0)$.

Proof. The closed linear hull of $\cup_{t \in[0,1]} P(t)$ is separable and contains all the contingent derivatives to $P$. Restricting everything to this subspace, all properties of $F$ remain valid, so for the sake of simplicity, we may assume that $X$ 
is separable. Take an integrable bound $\ell \in L^{1}[0,1]$ of $F$ such that $\ell(t) \geq 1$ for a.e. $t \in[0,1]$. Define $g=\int_{0} \ell$. In [21] we used a continuous linear injection $A: X \rightarrow L^{2}[0,1]$ such that for every weakly compact subset $W \subseteq X$ the set $A(W)$ is compact in $L^{2}[0,1]$, moreover if $W$ is compact, then $A$ is a homeomorphism between $W$ and $A(W)$. Now take the tube $A \circ P \circ g$ and the map

$$
G(t, y)=\frac{1}{\ell\left(g^{-1}(t)\right)} \cdot A\left(F\left(g^{-1}(t), A^{-1}(y)\right)\right) .
$$

Then $A \circ P$ and $G$ obviously satisfy the conditions of Proposition 2 (the values of $G$ are contained in the unit ball of $L^{2}[0,1]$ and $G$ is u.s.c. in the second variable), so we have that there is a function $y \in A C_{L^{2}}[0,1]$ which satisfies (1) with respect to $A \circ P$ and $G$. It is easy to verify that $x=A^{-1} \circ y \circ g$ is a solution to (1) with respect to $P$ and $F$.

\section{The tangent cone form of Kakutani's theorem}

Finally, as an other application of Theorem 3, we present a new proof to the equilibrium form of the classical Kakutani's fixed point theorem.

In the following, let $X$ be a Banach space. Let us assume the fixed point theorem: If $K \subseteq X$ is nonempty convex and compact set and $F: K \rightarrow \mathcal{P}(K)$ is a closed and convex valued u.s.c. map, then $F$ has a fixed point, i.e. a point $x \in K$ such that $x \in F(x)$.

Take a convex and compact subset $K$ in $X$. Then it is easy to see that the projection map

$$
\begin{aligned}
\Pi_{K} & : \quad X \rightarrow \mathcal{P}(K), \\
\Pi_{K}(x) & : \quad=\{y \in K:\|y-x\|=d(x, K)\}
\end{aligned}
$$

is convex compact valued and u.s.c. The next observation is also easy to verify: if $x \in K$ and $y \in X$, moreover

$$
y \in x+T_{K}(x) \text { and } x \in \Pi_{K}(y),
$$

then necessarily $x=y$.

Lemma 6 Let $f: K \rightarrow X$ a continuous function such that

$$
f(x) \in T_{K}(x)
$$

for every $x \in K$. Then there is an equilibrium point $x_{*} \in K$ of $f, i$. e.,

$$
f\left(x_{*}\right)=\mathbf{0} .
$$

Proof. Take the convex compact valued u.s.c. map

$$
\begin{aligned}
F & : \quad K \rightarrow \mathcal{P}(K), \\
x & \mapsto \Pi_{K}(x+f(x)) .
\end{aligned}
$$


Then by Kakutani's fixed point theorem (e. g. Theorem 1 in [22]) we get that $\exists x_{*} \in K$ such that

$$
x_{*} \in \Pi_{K}\left(x_{*}+f\left(x_{*}\right)\right)
$$

on the other hand,

$$
f\left(x_{*}\right) \in T_{K}\left(x_{*}\right) \text { i.e. } x_{*}+f\left(x_{*}\right) \in x_{*}+T_{K}\left(x_{*}\right),
$$

so from the previous observation we get that

$$
x_{*}=x_{*}+f\left(x_{*}\right),
$$

thus, $f\left(x_{*}\right)=\mathbf{0}$.

TheOREM 5 Let $F: K \rightarrow \mathcal{P}(X)$ be a nonempty convex and closed valued u.s.c. map such that

$$
F(x) \cap T_{K}(x) \neq \emptyset
$$

for every $x \in K$. Then there is an equilibrium point of $F$ i.e. a point $x_{*} \in K$ such that

$$
\mathbf{0} \in F\left(x_{*}\right) .
$$

Proof. Since $K$ is convex, the map $T_{K}: K \rightarrow \mathcal{P}(X), x \mapsto T_{K}(x)$ is nonempty convex and closed valued l.s.c. map. Then by Theorem 3 , we have that for all positive $\varepsilon$, there is a continuous function $f_{\varepsilon}: K \rightarrow X$ such that

$$
\operatorname{graph} f_{\varepsilon} \subseteq B(\operatorname{graph} F, \varepsilon) \quad \text { and } \quad f_{\varepsilon}(x) \in T_{K}(x)
$$

for every $x \in X$. From Lemma 6 , we get a point $x_{\varepsilon} \in K$ such that $\mathbf{0}=f_{\varepsilon}\left(x_{\varepsilon}\right)$, i.e. $\left(x_{\varepsilon}, \mathbf{0}\right) \in \operatorname{graph} f_{\varepsilon} \subseteq B(\operatorname{graph} F, \varepsilon)$, thus,

$$
(K \times\{\mathbf{0}\}) \cap B(\operatorname{graph} F, \varepsilon) \neq \emptyset
$$

for every $\varepsilon>0$, hence

$$
(K \times\{\mathbf{0}\}) \cap \operatorname{graph} F \neq \emptyset
$$

since $K \times\left\{0_{X}\right\}$ kis compact and graph $F$ is closed. This just means that there is a point $x_{*} \in K \operatorname{such}$ that $\left(x_{*}, \mathbf{0}\right) \in \operatorname{graph} F$ i.e. $\mathbf{0} \in F\left(x_{*}\right)$.

\section{References}

[1] AUBIN, J.-P.: Viability Theory, Birkhäuser, Boston, Basel, Berlin, 1991.

[2] AUBIN, J.-P. and CELLINA, A.: Differential Inclusions, Springer-Verlag, Berlin, 1984.

[3] AUBIN, J.-P., LYGEROS, J., QUINCAMPOIX, M., SASTRY, S., and SEUBE, N.: Impulse differential inclusions: A viability approach to hybrid systems, IEEE Transactions on Automatic Control, 47 (2002): pp. 2-20. 
[4] AUBIN, J.-P., BAYEN, A., and SAINT-PIERRE, P.: Viability Theory: New Directions. Springer-Verlag, Berlin, Heidelberg, 2011.

[5] BOTHE, D.: Multivalued differential equations on graphs, Nonlinear Anal. Th. Meth. Appl., 18 (1992) pp. 245-252.

[6] CAStaing, C. and VAlADIER, M.: Convex Analysis and Measurable Multifunctions, Lecture Notes in Math. 580, Springer-Verlag, Berlin, 1977.

[7] COLOMBO, G.: Weak flow-invariance for nonconvex differential inclusions, Diff. and Int. Equations, Vol. 5, No. 1 (1992), pp. 173-180.

[8] DEIMLING, K.: Multivalued Differential Equations, De Gruyter, Berlin, New York, 1992.

[9] DEIMLING, K.: Multivalued differential equations on closed sets, Differential and Integral equations, Vol. 1, No. 1 (1988), pp. 23-30.

[10] FRANKOWSKA, H., PLASKACZ S. and RZEŻUCHOWSKI, T.: Measurable viability theorems and Hamilton-Jacobi-Bellman equation, Cahiers de mathematiques de la decision, Ceremade, Univ. de Paris-Dauphine (1992).

[11] GAO, Y.: Viability criteria for differential inclusions, Journal of Systems Science and Complexity, Vol. 24, Issue 5 (2011), pp. 825-834.

[12] HAN, Y. and GAO, Y.: Determining the viability for hybrid control systems on a region with piecewise smooth boundary, Numer. Algebra Control Optim. Vol. 5, No. 1 (2015), pp. 1-9.

[13] GAVIOLI, A. and MALAGUTI, L.: Viable solutions of differential inclusions with memory in Banach spaces, Portugaliae Mathematica, Vol. 57, Issue 2 (2000), pp. 203-217.

[14] GONCHAROV, V. V.: On existence of solutions of some type of differential inclusions on a compact set, Sibir. Math. Journ. 31 (5) (1990) pp. 24-30 (in Russian)

[15] HADDAD, G.: Monotone trajectories of differential inclusions with memory, Israel J. Math. 39. (1981) pp. 83-100.

[16] KAKUTANI, S.: A generalization of Brouwer's fixed point theorem, Duke Math. J. 1941. 8. pp. 457-459.

[17] KÁNNAI Z. and SZABÓ I.: Viability theorems in Banach spaces, Pure Math. Appl. Ser. B 1 (1990) pp. 25-38.

[18] KÁNNAI Z. and TALLOS P: Viable trajectories of nonconvex differential inclusions, Nonlinear Anal. Th. Meth. Appl., 18 (1992) pp. 295-306.

[19] KÁNNAI Z. and TALLOS P: Viable solutions to nonautonomous inclusions without convexity, Central European Journal of Operations Research, (2003), pp. 47-55. 
[20] KÁNNAI Z.: Viability theorems on strongly sleek tubes, Annales Univ. Sci. Budapest Sect. Comp. 13 (1992) pp. 63-75.

[21] KÁNNAI Z.: Viability theorems with convex and weakly compact right-hand side, Pure Math. Appl. Ser. B Vol. 1 No. 4 (1990) pp. 241-249.

[22] KY FAN: Fixed-point and Minimax Theorems in Locally Convex Topological Linear Spaces, Proceedings of the National Academy of Sciences of the United States of America Vol. 38 (2) 1952. pp. 121-126.

[23] LEDYAEV, YU. S.: Criteria for viability of trajectories of nonautonomous differential inclusions, J. Math. Anal. Appl. 182, (1994) pp. 165-188.

[24] NAGUMO, M.: Über die Lage der Integralkurven gewöhnlicher Differentialgleichungen, Proc. Phys. Math. Soc. Japan 24 (1942) pp. 551-559.

[25] RZEŻUCHOWSKI, T.: Scorza-Dragoni type theorem for upper semicontinuous multivalued functions, Bull. Acad. Polon. Sci., Sér. Math. 28 (1980) pp. 61-66.

[26] TALLOS P: Viability problems for nonautonomous differential inclusions, SIAM. J. Control Optim. 29 (1991) pp. 253-263. 